\title{
The Value of Converged IT Infrastructures
}

\author{
Alberto Polzonetti ${ }^{1 *}$ \\ ${ }^{1}$ School of Technological Science, University of Camerino Italy, Camerino, Italy \\ *Alberto Polzonetti, E-mail: alberto.polzonetti@unicam.it
}

\begin{abstract}
This paper analyzes the economic value of implementing converged systems based on different research conducted by analyst firm last year, in particular: converged Infrastructure Platform in May 2014 and Trends in Private Cloud Infrastructure April. Particularly we discuss in first sections the value of converging solutions and the diffusion development of converged computing infrastructure. Finally we talk about the advantages of this solution with particular emphasis on management and economic aspect.
\end{abstract}

\section{Keywords}

converged systems, IT efficiency, systems management

\section{The Value of Converged Computing Solutions}

Converged computing systems are revolutionizing the way IT thinks about infrastructure investments. Delivered by both vendors and resellers, they combine hardware and software components in a unified package with benefits and advantages that far exceed Present Mode of Operations (PMO) frameworks. The results are enabling IT organizations to transform these legacy, internally built infrastructures into productive and economically efficient service centers. Converged platforms provide true value and economic advantages via a lower Total Cost of Ownership (TCO), a faster Return on Investment (ROI), and improved business agility. They also enable IT pros to work smarter on more strategic projects that advance business goals, as opposed to just "keeping the lights on". Converged platforms also complement the new generation of policy-based, software-defined infrastructures that are increasingly defining the rapidly changing world of corporate computing. It all adds up to financial benefits, IT operational efficiencies, enhanced system management from a single view, and a razor-sharp competitive edge.

\subsection{Management Efficiencies}

System management and IT automation are key components of any successful IT project, and infrastructure management is greatly enhanced with converged computing platforms where all the different element managers of servers, storage, and networking are collapsed, enabling IT operation teams to manage, monitor, report, and troubleshoot from a single pane of glass. This represents a huge benefit to beleaguered IT organizations that are constantly under pressure to do more with less, even as they battle on multiple fronts with runaway mobility demands, application development shortfalls, and 
the deluge of structured and unstructured big data flowing into their organizations. These management capabilities can enable automation capabilities across server, storage, and networking and drive exponential operational efficiencies. While the individual components that complete a converged computing platform are important, it's the automation capabilities that drive the long-term benefits and success of the investment. This includes basic inventory, compliance checking, and the ability to apply patches and updates in an automated fashion with confidence that the entire system will respond positively to any changes.

In this pressure-packed environment, the value proposition for converged computing systems is appealing to a rapidly expanding number of CIOs and other senior IT executives who are tired of dealing with the never-ending need to refresh their traditional IT infrastructures so they can stay on top of the enterprise-wide projects underway at their organizations. The value of the integration along with the economic benefits of this approach and the ease of system management are causing more companies to embrace converged computing platforms, making them a standard that will continue to grow over time. In fact, according to the aforementioned research, $88 \%$ of respondents reported being extremely satisfied or satisfied with their converged system solution and $74 \%$ percent said that they would be extremely likely or likely to recommend it to a peer.

\subsection{The Value of Integration and Operational Agility}

The value of converged systems begins with IT agility and integration, including testing and sizing, which save time and decrease OpEx and risk. The hardware and software components are integrated into a single, consumable IT system that is constructed before it is delivered to the business. This system unites the independent pieces of IT infrastructure, which are normally operated separately, through policy and common functionality driven by management software. That software then helps automate IT processes to form simplified computing platforms that are workload-optimized for virtualization, cloud computing, and big data.

The converged system's beauty is found in its simplicity for the business and benefits returned on an annual basis. The pre- configured, pre- validated solution aggregates commonly deployed IT infrastructure components - servers, storage, networking, virtualization, and software-into prepackaged systems delivered directly from the vendor or reseller channel for streamlined consumption measured in hours or days. Based on best practices, these systems combine hardware integration, software orchestration, and, under certain circumstances, specific engineering and configuration into holistic rack architectures designed to operate harmoniously.

In addition to accelerating time to value and reducing both cost and risk, converged computing systems also offer a single-vendor support model, and the ability to scale in a flexible, modular fashion beyond the organization's initial investment and throughout the lifecycle of the investment. This simply cannot be achieved with PMO deployment models without massive investments in IT staff resources, expertise, and time. 


\subsection{Economic Advantages}

Inefficient data center environments littered with a lengthy list of mundane IT tasks and lack of IT process automation are exhausting IT's time and budgets. This is causing businesses to struggle with embracing new technology innovations that could improve IT service delivery and lower the cost of operations. In order for technology to become strategic - rather than tactical—when it comes to business agility and innovation, companies must strive for a more balanced approach that focuses on both economic imperatives to maintain existing IT environments and IT operational efficiencies.

ESG's research provides further evidence that businesses are recognizing a return on their investment as exemplified by the positive remarks made by respondents when asked how they would evaluate the TCO of their organizations' convergent computing platforms. Sixty-six percent reported their TCO outcomes as either excellent or good.

In order to accurately measure the success of any project and justify its investment, IT must be aware of its impact and have a firm grasp on the metrics needed to monitor and compare the project's performance with existing operations. Converged systems are poised to pass this test, and can provide numerous benefits that cost-justify their acquisition and implementation.

These benefits include:

1) Quicker ROI-Evidenced by efficiency gains from faster time to value, competitive acuity through greater business agility, and more strategic use of IT staff.

2) Lower TCO-Evidenced by considerable annual benefits including reduced time to production, decreased time spent on management and maintenance, and increased uptime.

3) Risk reduction-A quantifiable, key benefit that loosely structured PMO integration projects do not always achieve, which can lead to time-consuming failures and costly remediation. By way of comparison, converged systems follow a more certain path because they are designed to minimize variables and work right out of the box.

4) Agility —increases the ease of deploying additional capacity throughout the lifecycle of the system.

\section{Increasing Usage of Converged Computing Systems}

There is no doubt that the value of consuming converged computing infrastructure is building momentum in the IT community. In order to assess just how strongly the converged system value proposition is resonating, and how rapidly it is leading to deployments, ESG asked survey respondents about their organizations' usage of and interest in converged computing technology, defined as platforms in which servers, storage, network connectivity, and software are combined in a single solution. Data from that research reveals that $33 \%$ of organizations have already deployed converged computing platforms, while an additional $44 \%$ have plans for or interest in these types of solutions. Not many IT projects can go live with the simple flip of a switch, but IT organizations are showing excellent progress with their private cloud initiatives, which are workload-optimized for virtualization. In order to continue and improve their progress with these initiatives, IT must evaluate the composition 
of their underlying private cloud infrastructures to determine how they will support pooled server, storage, and networking resources that are driven by a common management layer, which must support rapid adaptability to change. IT organizations that couple converged system deployments with their private cloud initiatives have the unique opportunity to experience these synergies and accelerate their progress within an infrastructure that is designed and certified to meet the requirements of private cloud while offering a reliable foundation and a predictable infrastructure investment.

\subsection{Time to Value}

Among the many benefits of converged computing platforms, time to value stands out prominently. Rather than relying on the purchase of compute, storage, and networking from three separate vendors and channel partners, converged infrastructures simplify the deployment process by streamlining system planning, design, procurement, setup, configuration, and testing, in addition to simplifying application staging, management, and ongoing maintenance.

Time to value is comprised of these claims that vendors communicates to its customer base:

1) Time from order to operations - according to Converged Systems, the time from order to operations has been pushed down to as few as 15 days.

2) Time to additional capacity - the systems come converged and pre-configured, and can be added instantly. Normally, the vendor is making commitments to its customers on delivering the capacity in five days.

Together, these benefits provide significant efficiency gains that directly contribute to expedite ROIs, and are a major reason why IT organizations are rapidly adopting this type of infrastructure.

ESG research validates that it doesn't take long for organizations to install and configure converged systems. The bar has moved from weeks or months with traditional PMO infrastructures to days. The research indicates that $92 \%$ of respondents were able to install and configure their system within five days. 


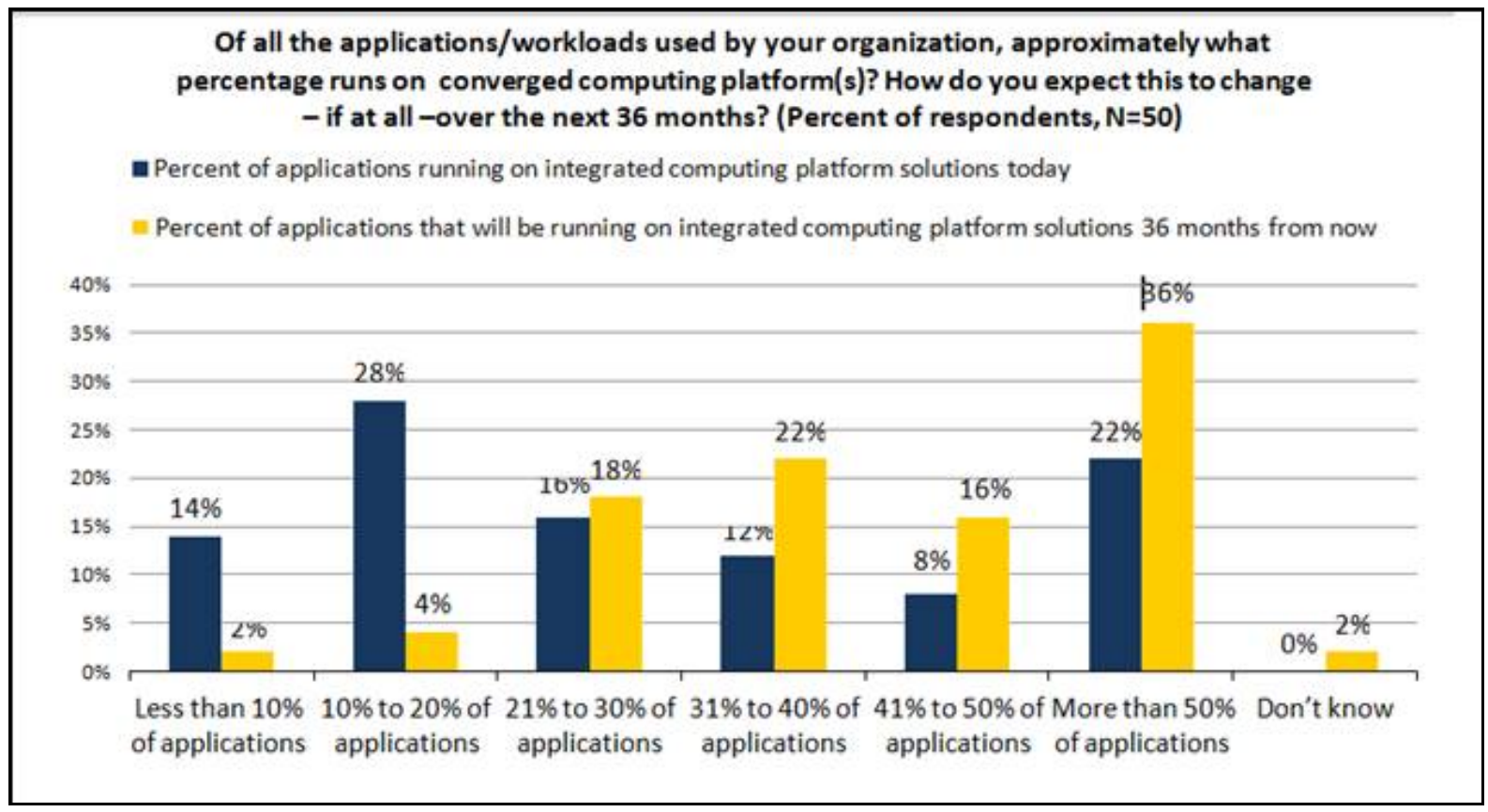

Figure 1. Percentage of Applications/Workloads Running on Converged Computing Platforms, Now and in 36 Months (Source: Enterprise Strategy Group, 2014)

\subsection{Decision Time}

Faced with the enticing possibility of bringing converged computing systems online in days instead of weeks and months, companies have two choices to consider: choice one is committing to a converged computing platform, and choice two is taking the traditional approach of building the IT infrastructure in house.

Committing to a converged system creates the short-term advantage for organizations of having a phalanx of tightly converged, fully optimized, cutting-edge hardware and software products at their disposal. More importantly, it also creates a long-term advantage where companies can quickly and flexibly upgrade their systems with pre- configured components created specifically for them. Simply maintaining the individual pieces of infrastructure can grind IT to a halt when patches, drivers, and firmware updates have to be applied. With a converged system, the updates are applied at the system level, enabling IT to validate that the full system will stay in sync. If your company is locked in a tight, highly competitive market with other players who are deploying convergent strategies, how can you afford not to follow suit?

Let's look at the situation from another perspective. Choice two is taking the traditional, PMO path to technology refresh, which involves many IT teams congregating and determining what their infrastructure looks like. The chances are that it will also involve disparate procurement strategies for each major component-compute, network, storage-adding to the complexity of the actual procurement and implementation processes. IT organizations can take this risky, time-consuming approach, or they can have a vendor do all the heavy integration lifting at the factory, deliver a 
validated system, and implement it within a matter of days. Hello, time to value!

\subsection{Organizations Plan to Expand Deployment}

Delving deeper into businesses' momentum for adopting converged systems, ESG asked respondents to indicate approximately what percentage of their applications and workloads currently run on converged computing platforms, and how they expect that situation to change over the next three years. The results show that although $28 \%$ of respondents are running $10-20 \%$ of their applications and workloads on converged infrastructures today, within three years, $36 \%$ will be running more than $50 \%$ of their apps on converged computing platforms. These numbers paint a picture of a significant shift and growth both in the number of organizations using converged systems as well as their level of usage. This momentum is building now and will be hitting its stride in the near future.

\section{Favorable Economics Drives Investments}

So what motivates IT organizations to deploy—or consider deploying — converged platforms? While the initial set-up process and value of integration performed at the factory is important, especially in more dynamic or elastic environments, the economic impact of these investments over the lifecycle of the investment is also a key consideration. It follows then that when pressed to identify the primary reason behind converged computing platform deployment decisions, nearly one in five current users singled out improved total cost of ownership - the topmost reason organizations are deploying converged systems. TCO was followed closely by increased agility of virtual machine provisioning $(14 \%)$, improved scalability (10\%), ease of management (10\%), and speed of deployment $(8 \%)$ as the top five primary reasons for deploying converged systems.

Despite the perceptions of some who believe converged IT infrastructures require significant capital outlays, many organizations are looking at the bigger picture and realizing the potential profitable impact these solutions can have in terms of improved time to value and operational efficiency during their lifecycles. 


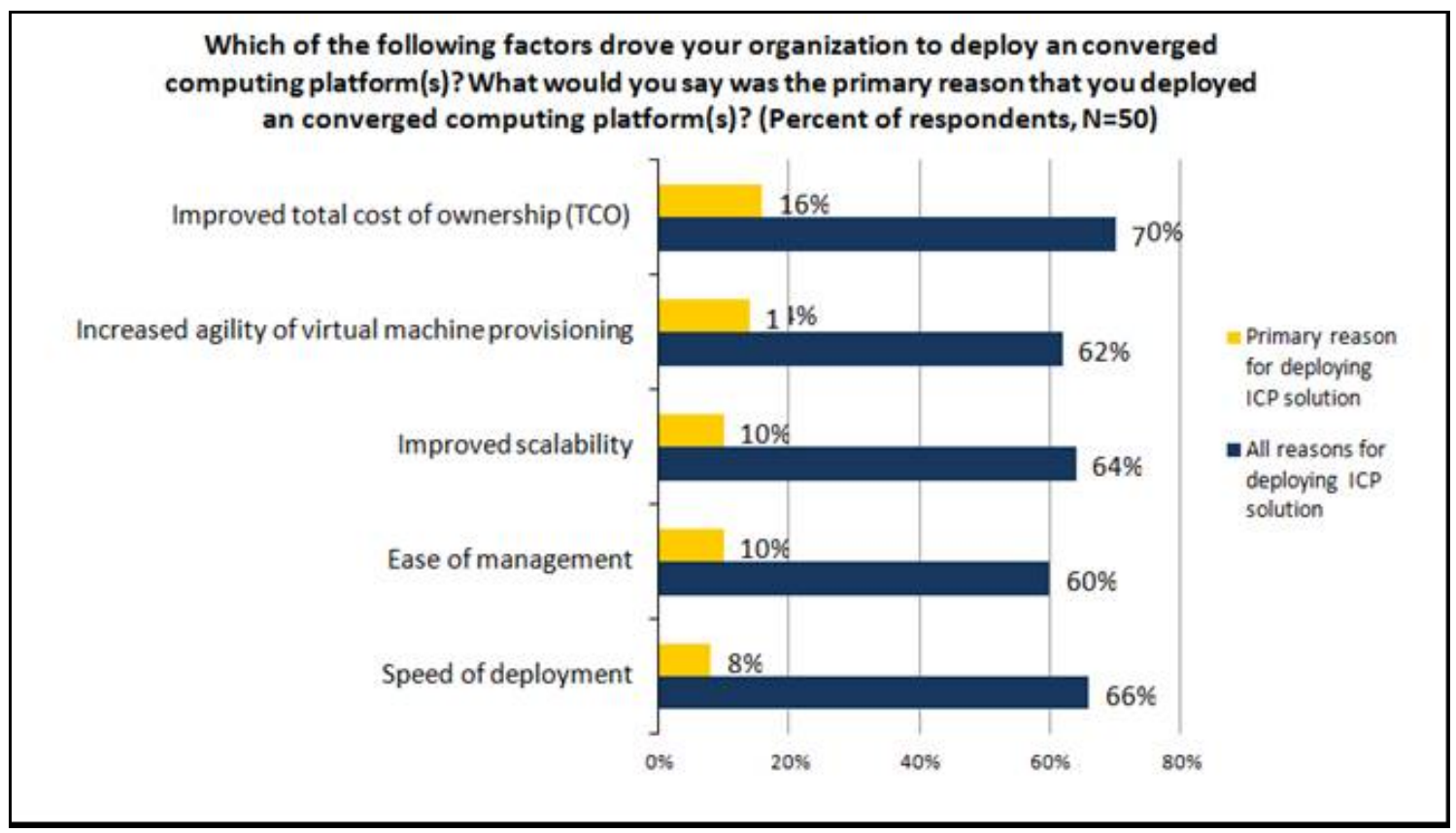

Figure 2. Top Five Factors Driving Converged Computing Platform Deployment (Source:

Enterprise Strategy Group, 2014)

\section{Optimizing IT Operations Efficiencies}

As previously mentioned, IT organizations are looking at converged computing platforms as an opportunity to consolidate individual element managers into an abstraction layer that provides a single point of management. This serves to simplify ongoing management requirements, which creates the potential for the reallocation of IT headcount from tactical supervision of systems to more strategic endeavors, such as data analytics initiatives.

In this environment, procurement of server, storage, networking, software, and management within a converged solution can help minimize interoperability testing and technology integration, reduce complexity, and streamline IT operations. In addition to the improved system performance and uptime that result from hardware compatibility, converged solutions also simplify data center administration through automation and management capabilities.

Automation plays a key role and provides added value in many system-wide processes because once a template is created for a process, it can be repeated many times, avoiding common errors, lowering risk, and without requiring IT intervention.

Rather than operating as discrete server, storage, and networking silos, convergent infrastructures break down those silos and pool resources across systems. These resources are then automatically allocated both within and between racks based on workload demands and performance requirements, which prevents IT from having to over-provision resources for peak workloads. 


\subsection{Ease of Management}

Ease of management is a mantra among many IT organizations. The vast majority of those organizations $(76 \%)$ believe that converged solutions are easier to manage than device-centric management solutions traditionally associated with computer, networking, and storage components. Simplified management drives such benefits as faster provisioning times, improved visibility and reporting, compliance adherence and risk mitigation, and positive organizational behavior. ESG firmly believes that these research results reflect an irreversible migration to converged IT infrastructures that will be compatible with industry-wide, policy-based, software-defined architectures.

The case for converged systems only becomes more compelling as additional ESG research results are factored into the equation. This is evidenced by the impressive array of specific management-related benefits that organizations have realized. Starting with a quick litany of benefits: streamlined IT operations, reduced troubleshooting for IT staff, and IT process automation, users can quickly identify and remediate issues, prompting their converged systems to positively respond to new policy settings by automating common tasks.

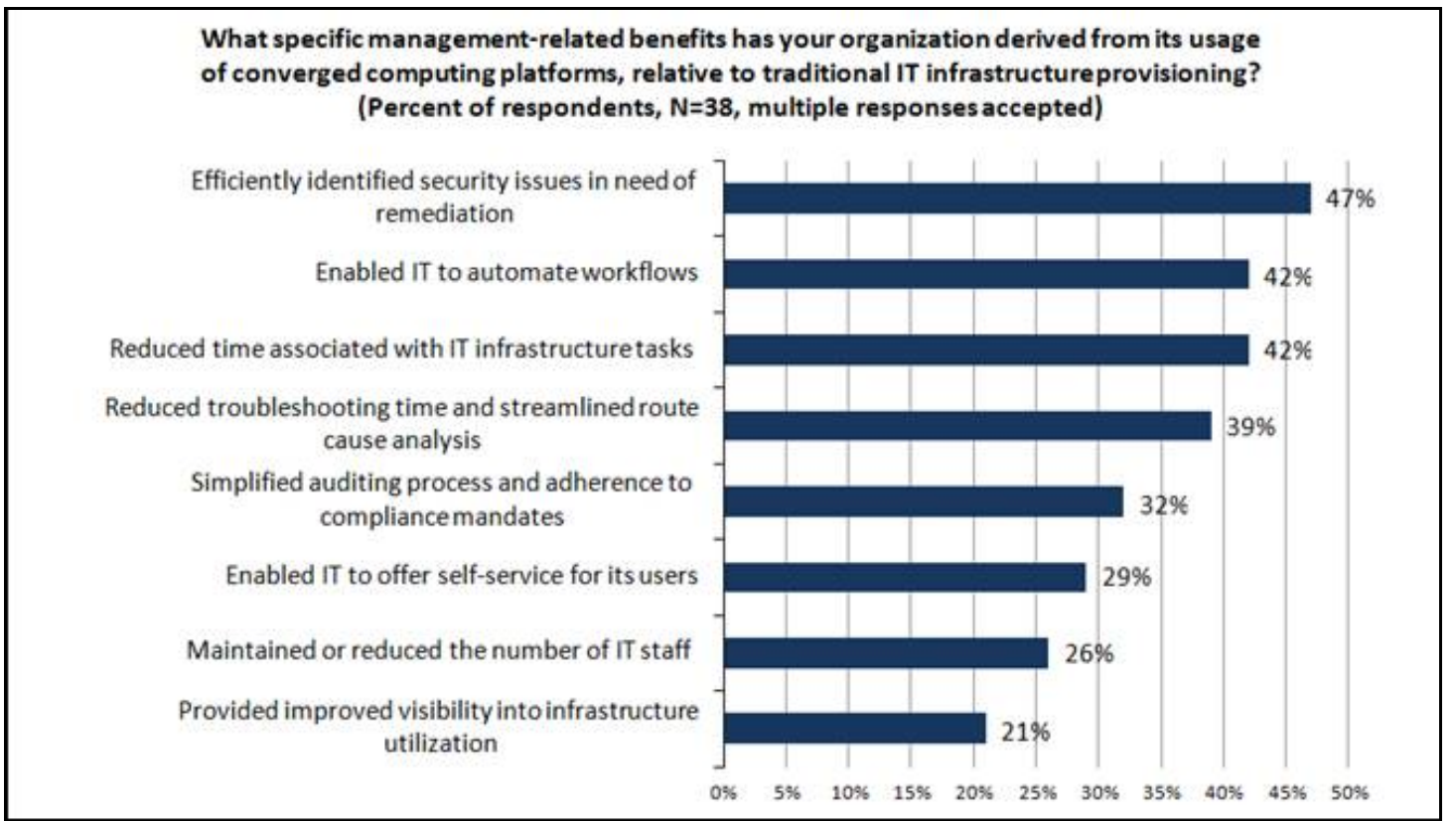

Figure 3. Management-Rlated Benefits Derived from Converged Computing Platforms

\section{Deployment (Source: Enterprise Strategy Group, 2014)}

\subsection{Convergence in the Cloud}

The investment in converged systems introduces improved efficiency and lays a solid foundation for cloud consumption. Now that the cloud has significantly evolved, the value of consolidated management functionality is further evidenced in market-leading cloud service management platforms that federate across on-premises and off-premises public cloud resources. These cloud platforms offer 
automated end-to-end lifecycle management for any cloud service to optimize infrastructure and monitor applications. It is important to recognize the value of this management model and how it can be leveraged for current and planned orchestration investments. The initial investments that provide a foundation for infrastructure automation are key decisions toward adopting a hybrid cloud model that enables IT to manage and automate IT processes across a variety of workloads and use cases.

\section{Conclusions}

The pace of change driven by converged systems and highly integrated IT infrastructures is remarkable. It wasn't that long ago that the kinds of benefits we're realizing now were more pie-in-the-sky than they were feet-on-the-street. It seems like overnight we have jumped from loosely confederated legacy systems based on point technologies - such as servers, networking, and storage- to tightly bundled, automated configurations that are actually doing more with less human intervention. These days, it seems, the lights don't need to be literally turned on in order for a company to be productive.

The value of this integration is found in an IT vendor's ability to automate tools and services across the hardware and software associated with these point technologies into a single system that is pre-integrated and pre-tested for customers before they receive it. The allure-the value - of this simple approach is undeniable to CIOs and other senior IT executives who find themselves not only constantly struggling to keep up with the pace of change that characterizes their current IT infrastructures, but also concerned about their ROI on present-day legacy systems as well.

The economic advantages of converged platforms are revealing themselves at an opportune time when inefficient data centers are turning into cost centers that consume IT time and money. However, despite the bountiful benefits of these advanced platforms, such as increased ROI, reduced TCO, and expedited time to value, significant financial gains can only be achieved through careful planning, which includes balancing the need for operational efficiencies with real-world economic imperatives. The burden is on savvy users to prove the value of their converged infrastructures.

Invaluable tools such as policy-based automation and easy-to-use management software maximize those operational efficiencies. Policy-based automation, in particular, is integral to the success of converged systems because it frees up IT professionals to perform other tasks that require their highest levels of expertise. Without a doubt, the research has shown that there's a lot of recognized value to be brought by these converged computing platforms. While organizations are gaining some initial traction with them today, their aggressive plans over the next few years ensure that these converged systems will be the de facto standard of the future.

\section{References}

Bannazadeh, H., Leon-Garcia, A., Redmond, K., Tam, G., Khan, A., Ma, M., ... Chow, P. (2011). Virtualized application networking infrastructure. In Testbeds and Research Infrastructures, Development of Networks and Communities (pp. 363-382). Springer Berlin Heidelberg. 
Ghose, A. (April, 2013). The 1st International Workshop on Self-Managing Pervasive Service Systems (SeMaPS, 2012). In Service-Oriented Computing-ICSOC Workshops 2012: ICSOC 2012, International Workshops ASC, DISA, PAASC, SCEB, SeMaPS, and WESOA, and Satellite Events, Shanghai, China, November 12-15, 2012, Revised Selected Papers (Vol. 7759, p. 253). Springer.

ESG Custom Research. (May, 2014). Converged Infrastructure Platforms.

ESG Research Report. (April, 2014). Trends in Private Cloud Infrastructure.

Falfushinsky, V., Skarla, O., \& Tulchinsky, V. (2014). Integration of Cloud Computing Platform to Infrastructure. International Journal of Computing, 12(4), 333-339.

Uddin, M., Shah, A., \& Rehman, A. (2014). Metrics for Computing Performance of Data Center for Instigating Energy Efficient Data Centers. Journal of Scientific \& Industrial Research, 73, 11-15. 